\title{
A Fiery Pivot
}

\author{
R Cole Pueringer, MD ${ }^{1,2}$, Marshall Mazepa, MD 3,4 , Andrew PJ Olson, MD $4,5,6,7,8$, Samuel T Ives, MD*
}

This icon represents the patient's case. Each paragraph that follows represents the discussant's thoughts.

'Division of General Internal Medicine, Department of Medicine, Hennepin County Medical Center, Minneapolis, Minnesota; ${ }^{2}$ Division of Medical Toxicology, Department of Emergency Medicine, Regions Hospital, Saint Paul, Minnesota; ${ }^{3}$ Division of Hematology, Oncology and Transplantation, University of Minnesota, Minneapolis, Minnesota; ${ }^{4}$ Department of Medicine, University of Minnesota, Minneapolis, Minnesota; ${ }^{5}$ Division of General Internal Medicine, Minneapolis, Minnesota; ${ }^{6}$ Division of Pediatric Hospital Medicine, University of Minnesota, Minneapolis, Minnesota; ${ }^{7}$ Department of Pediatrics, University of Minnesota, Minneapolis, Minnesota; ${ }^{8}$ University of Minnesota Medical School, Minneapolis, Minnesota.

A 62-year-old man with metastatic non-small cell lung cancer (NSCLC) presented to the Emergency Department with 3 days of progressive generalized weakness, anorexia, and nonbloody diarrhea. He denied fever, chills, nausea, vomiting, cough, shortness of breath, or abdominal pain. He had no sick contacts.

One diagnostic approach for patients with cancer who present with new symptoms is to consider diagnoses both related and unrelated to the cancer. Cancer-related diagnoses can include the broad categories of complications related to the tumor itself (such as mass effect), paraneoplastic phenomena, or treatment-related complications (such as infection from immunosuppression or chemotherapy toxicity).

For this patient with metastatic NSCLC, weakness, anorexia, and diarrhea are unlikely to be related to mass effect unless the patient has peritoneal metastases (an uncommon complication of NSCLC) with carcinomatosis-associated diarrhea.

Paraneoplastic phenomena, such as hypercalcemia or hyponatremia from the syndrome of inappropriate antidiuretic hormone (SIADH), are common with NSCLC and could both lead to weakness and anorexia. Hematologic consequences of NSCLC (or its treatment) include anemia, thrombosis, and thrombotic microangiopathy (TMA), though diarrhea, in the absence of abdominal pain or hematochezia, would be unexpected.

Weakness, anorexia, and diarrhea may also be symptoms of chemotherapy toxicity or an infection resulting from immunosuppression. It would be important to know what specific treatment the patient has received. Chemotherapy commonly causes neutropenia and predisposes to rapidly progressive infections, while immunotherapies have other toxicities. Diarrhea is a common toxicity of the checkpoint inhibitors and anaplastic lymphoma kinase (ALK) inhibitors that are frequently used to treat metastatic NSCLC. Checkpoint inhibitors also are known to cause a wide range of autoimmune phenomena including colitis.

*Corresponding Author: Samuel T. Ives, MD; Email: Samuel.ives@hcmed.org. Published online first June 17, 2020.

Received: July 25, 2019; Revised: January 28, 2020; Accepted: January 31, 2020 (๑) 2020 Society of Hospital Medicine DOI 10.12788/jhm.3390
Finally, the patient's symptoms may be unrelated to the cancer. Weakness, anorexia, and nonbloody diarrhea could be signs of viral or bacterial gastroenteritis or Clostridioides difficile colitis particularly with frequent healthcare contact or antimicrobial use.

Two days prior, he had been diagnosed with nonsevere Clostridioides difficile colitis in an acute care clinic. He was started on oral metronidazole, but his diarrhea worsened over the next day and was accompanied by weakness and anorexia. Additional past medical history included untreated hepatitis $C$ infection, chronic kidney disease stage 3 , seizure disorder, and left lung NSCLC (adenocarcinoma). The lung cancer was diagnosed 8 months prior when he had presented with hemoptysis and 3 months of progressive constitutional symptoms. Imaging at that time revealed metastases to the contralateral lung and regional lymph nodes, as well as vertebrae, ribs, and pelvis. He had no abdominal metastases. He was initially treated with carboplatin and paclitaxel. After a partial response to initial chemotherapy, he developed peripheral neuropathy and was switched to gemcitabine 12 weeks ago. He received five cycles of gemcitabine over 10 weeks. He was last administered gemcitabine 2 weeks prior. He had not received any additional chemotherapy or immunotherapy. He had a 40 pack-year history of smoking, but quit when diagnosed with cancer. He did not drink alcohol. $\mathrm{He}$ had no recent travel or sick contacts. He was not on any medications. He was homeless but staying with family members in the area. Additional review of systems was negative for recent bleeding, bruising, hemoptysis, melena, hematochezia, or hematuria.

Recent treatment with gemcitabine could contribute to the presentation in a number of ways. First, gemcitabine is associated with myelosuppression and neutropenia that could predispose him to infectious colitis. Second, gemcitabine is known to cause anemia, anorexia, diarrhea, and fatigue. Third, gemcitabine may also cause renal injury that can contribute to worsening anemia. He may be at greater risk of anemia and renal toxicity because of preexisting chronic kidney disease. Finally, gemcitabine can rarely cause TMA with characteristics that mimic the hemolytic-uremic syndrome with microangio- 


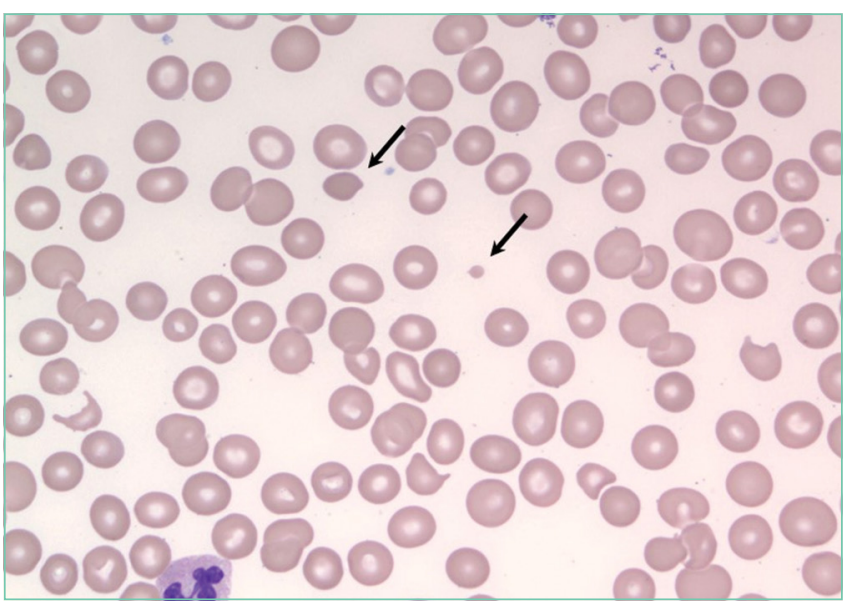

FIG 1. Initial peripheral blood smear is shown; arrows indicate pyropoikilocytes.

pathic hemolytic anemia, mild thrombocytopenia, and severe acute kidney injury (AKI).

In addition, worsening infectious colitis could certainly explain his presenting symptoms. At this point, local mass effect seems unlikely despite his metastatic disease. Lastly, it should be noted that, in an immunosuppressed cancer patient, multiple problems could be present at the same time. Laboratory testing should evaluate for hypercalcemia, SIADH, hematologic indexes, and renal function. If initial laboratory evaluation is unrevealing, abdominal imaging may be needed to assess for carcinomatosis, complications from colitis, typhlitis, abscess, or perforation.

On physical examination, the patient appeared fatigued. His temperature was $36.8^{\circ} \mathrm{C}$, blood pressure $158 / 72 \mathrm{~mm} \mathrm{Hg}$, pulse 88 beats per minute, respiratory rate 16 breaths per minute, and oxygen saturation was $96 \%$ while breathing ambient air. There was neither scleral icterus nor conjunctival injection but he had mild conjunctival pallor. Cardiovascular and lung examinations were normal. Abdominal exam revealed normal bowel sounds without tenderness or organomegaly. He had no supraclavicular, axillary, or inguinal lymphadenopathy. He was alert and oriented. Cranial nerves II through XII were intact. He had decreased muscle bulk in his extremities without focal weakness. Gait and reflexes were not tested.

Initial laboratory testing revealed a white blood cell count of $5.5 \mathrm{~K} / \mathrm{mm}^{3}$, hemoglobin of $5 \mathrm{~g} / \mathrm{dL}$ (hemoglobin 1 month prior was $10.1 \mathrm{~g} / \mathrm{dL}$ ), and platelet count of $20 \mathrm{~K} / \mathrm{mm}^{3}$ (platelet count 1 month prior was $246 \mathrm{~K} / \mathrm{mm}^{3}$ ). Creatinine was $3.9 \mathrm{mg} / \mathrm{dL}$ (compared with a baseline of $1.8 \mathrm{mg} / \mathrm{dL}$ ), and blood urea nitrogen was $39 \mathrm{mg} / \mathrm{dL}$. His sodium was $137 \mathrm{mEq} / \mathrm{L}$, potassium $4.2 \mathrm{mEq} / \mathrm{L}$, chloride $105 \mathrm{mEq} / \mathrm{L}$, bicarbonate $22 \mathrm{mEq} / \mathrm{L}$, and thyroid stimulating hormone $0.9 \mathrm{mU} / \mathrm{L}$. His total protein was $4.9 \mathrm{~g} / \mathrm{dL}$, albumin $2.1 \mathrm{~g} /$ $\mathrm{dL}$, alkaline phosphatase $60 \mathrm{IU} / \mathrm{L}$, alanine aminotransferase $17 \mathrm{IU} / \mathrm{L}$, aspartate aminotransferase $60 \mathrm{IU} / \mathrm{L}$, direct bilirubin $0.2 \mathrm{mg} / \mathrm{dL}$, and total bilirubin $0.5 \mathrm{mg} / \mathrm{dL}$. A chest $\mathrm{x}$-ray showed no infiltrates.

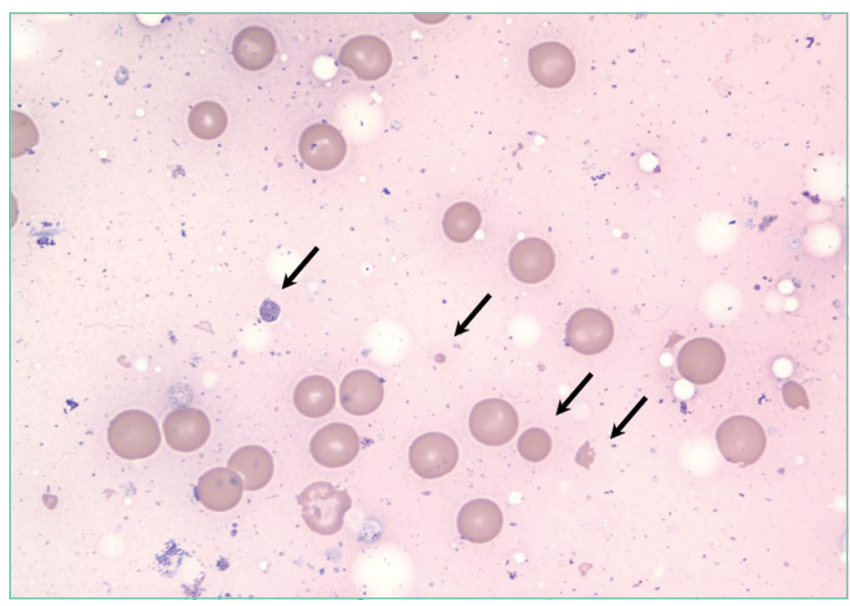

FIG 2. Bone marrow biopsy is shown: Touch imprint shows heat damage in RBCs caused by the friction of twisting the coring needle; arrows indicate pyropoikilocytes.

The patient's laboratory tests reveal several important new findings, including severe acute on chronic anemia, acute thrombocytopenia, and AKI, without clinical evidence of acute blood loss. These changes could be parts of a syndrome or multiple independent disorders. The most urgent priority is to evaluate for TMAs, many of which are fatal if not diagnosed and treated expeditiously. This includes thrombotic thrombocytopenic purpura (TTP), disseminated intravascular hemolysis (DIC), and atypical hemolytic uremic syndrome (aHUS). A manual review of a peripheral blood smear is required to evaluate for fragmented red blood cells (schistocytes). Thereafter, ancillary testing to confirm intravascular hemolysis would include measuring free plasma hemoglobin and lactate dehydrogenase (LDH). Additionally, in intravascular hemolysis, haptoglobin should be depleted and urinalysis should show heme-positive urine without RBCs. In this case the patient's normal bilirubin studies argue against hemolysis; however, elevated bilirubin is variably present in hemolytic anemias depending on the liver's ability to conjugate and excrete bilirubin, the relative degree of RBC turnover, and type of hemolysis. Patients with intravascular hemolysis lose hemoglobin directly into the urine leaving relatively little hemoglobin to be incorporated into bile once it has reached the reticuloendothelial system. This results in relatively normal bilirubin levels. More specific indicators of intravascular hemolysis include pink colored plasma on visual inspection (commonly done in the blood bank as part of assessing for hemolytic transfusion reactions), measuring plasma free hemoglobin, or by detecting hemoglobin in the urine.

If microangiopathic hemolytic anemia (MAHA) is excluded, then other causes of these laboratory abnormalities should be considered. Bleeding is the most common cause for anemia, and thrombocytopenia predisposes patients to bleeding. However, there is no evidence of bleeding in this patient, and such a rapid acute anemia is unlikely to be caused by occult blood loss alone. Concurrent anemia and thrombocytopenia could be evidence of bone marrow toxicity from chemotherapy or neoplastic infiltration. With marrow infiltration, there are 


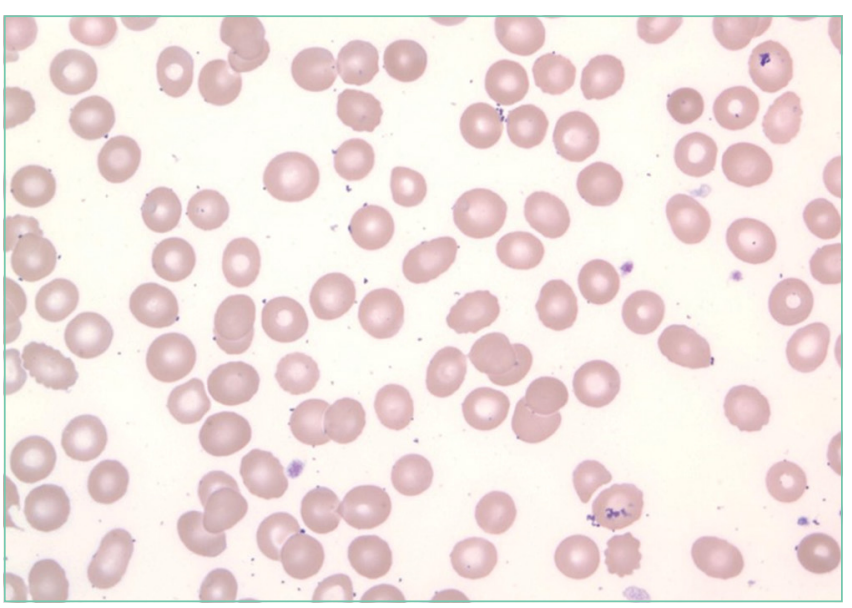

FIG 3. Three-month follow-up peripheral blood smear without pyropoikilocytes is shown.

typically signs on the peripheral smear of leukoerythroblastosis, with circulating nucleated red blood cells and early myeloid forms. Concurrent immune thrombocytopenia (ITP) and autoimmune hemolytic anemia (AlHA), or Evans' Syndrome, should also be considered. AlHA would be suggested by spherocytes on the peripheral smear, elevated LDH and a positive direct antibody test (DAT).

Regarding the AKI, the patient has diarrhea, which could lead to prerenal azotemia and acute tubular necrosis. A formal urinalysis would evaluate for prerenal and intrinsic kidney disease. TMA can cause intrinsic kidney injury with a benign urinary sediment. The blood urea nitrogen-to-creatinine ratio is not elevated, but in a patient with malnutrition this may not indicate prerenal azotemia. In summary, to differentiate potential TMAs from other causes, the patient needs a blood smear, coagulation studies, and an evaluation for hemolysis, including a urinalysis for free heme and any evidence of intrinsic kidney disease.

Urinalysis showed amber-colored, dilute urine with no white blood cells, red blood cells, protein, or casts. It was positive for blood and negative for bilirubin and hemosiderin. LDH was 1,382 IU/L (reference range 135-225 IU/L), and haptoglobin was unmeasurably low. His ferritin was $2,267 \mathrm{ng} / \mathrm{mL}$, serum iron was $57 \mathrm{mcg} / \mathrm{dL}$, total iron-binding capacity was $241 \mathrm{mcg} / \mathrm{dL}$, and transferrin was $162 \mathrm{mcg} / \mathrm{dL}$. Reticulocyte count was 6\% (reticulocyte index of 0.86 ). Vitamin B12 level was normal. DAT was negative; INR and aPTT were normal. Fibrinogen was $287 \mathrm{mg} / \mathrm{dL}$ (reference range 200-400 mg/dL), and D-dimer was 5,095 ng/mL (reference range $0-229 \mathrm{ng} / \mathrm{mL}$ ).

The urinalysis shows no active sediment to suggest vasculitis or glomerulonephritis. The kidney injury could be the result of renal toxicity from free hemoglobin or as part of TMA caused by microvascular thrombosis. The dilute urine makes prerenal azotemia less likely.

There is clearly acute intravascular hemolysis occurring as ev- idenced by hemoglobinuria, very high LDH, and undetectable serum haptoglobin. The hemolysis is acute because chronic intravascular hemolysis would lead to positive urine hemosiderin via deposition in the renal tubules. Autoimmune hemolytic anemia is much less likely, but not ruled out, by a negative DAT.

This syndrome can be further refined from acute anemia to acute anemia with likely nonimmune intravascular hemolysis, acute thrombocytopenia, and AKI with hemoglobinuria and a bland urinary sediment. At this point, intravascular hemolysis and kidney injury could be part of a unifying diagnosis. However, this does not account for the patient's thrombocytopenia, and TMA remains the best explanation for the constellation of findings. Review of the peripheral blood smear is urgent because evidence of MAHA would prompt urgent plasma exchange based on presumptive diagnosis of acquired TTP to later be confirmed with ADAMTS13 activity testing. Most TMAs are treated with supportive care only; TTP and aHUS have specific interventions that change the natural history of the disease (plasma exchange and anticomplement therapy, respectively). Given both the deadly natural history and opportunity to intervene with plasma exchange, patients with TMA should be treated with urgent plasma exchange until ADAMTS13 deficiency is confirmed or refuted. One TMA that can be excluded at this point is DIC. DIC in its acute and chronic forms nearly universally causes MAHA, thrombocytopenia, and consumptive coagulopathy including hypofibrinogenemia.

If MAHA is excluded, then other causes of intravascular hemolysis should be considered, along with causes of thrombocytopenia that might be occurring concurrently. Intravascular hemolysis can be further differentiated by etiologies primarily related to the RBC or whether the RBC is the innocent bystander amidst a systemic illness. RBC disorders include syndromes affecting RBC fragility like hereditary spherocytosis or RBC enzymopathies (G6PD deficiency), but these do not cause thrombocytopenia. One exception is an acquired membrane defect, paroxysmal nocturnal hemoglobinuria $(\mathrm{PNH})$, in which RBCs and other blood cells become susceptible to complement-mediated lysis. Testing for PNH by peripheral blood flow cytometry should be considered if the blood film lacks schistocytes. Systemic disorders that cause intravascular hemolysis include severe burns (heat damage to RBCs), RBC trauma from "march hemoglobinuria" or mechanical heart valves, immune (antibody-mediated) hemolysis from Rh immune globulin administration, cold agglutinin disease or $\mathrm{ABO}$ mismatched transfusion, and infections including the intraerythrocyte parasites malaria, Bartonellosis, and Babesiosis, as well as organisms that induce RBC fragility such as Leishmaniasis, Clostridium perfringens, and Haemophilus influenzae B.

On review of additional history, the patient had not recently received blood products. He had received heparin during prior hospitalizations, but had no prior history of thrombosis. He had no history of tick exposure. Peripheral blood smear was obtained and reviewed by a hematopathologist. It showed no schistocytes or spherocytes, but demonstrated pyropoikilocytosis. 
The blood smear helps narrow the differential further. The lack of schistocytes makes TMA far less likely and so plasma exchange is not urgently indicated. The differential still includes druginduced TMA (gemcitabine being a well-known cause for TMA) and cancer-associated TMA could still cause these findings, but plasma exchange does not improve outcomes. Acquired (immune) TTP is very unlikely unless the patient did not improve with supportive care or developed neurologic symptoms. Similarly, atypical (complement-driven) HUS would only be considered if renal failure did not improve with supportive care.

The blood smear does show a surprising finding of pyropoikilocytosis. Pyropoikilocytosis refers to changes in RBC shape (poikilocytosis) typically seen with thermal injury or rare RBC membrane structural defects. Hereditary pyropoikilocytosis, a very rare disease, is characterized by chronic hyperproliferative, compensated anemia, and occasional hemolytic crises. These crises are associated with splenomegaly, reticulocytosis, and elevated bilirubin with jaundice. As the patient has no history of similar episodes, the blood smear changes are not due to a hereditary cause and obviously not due to thermal injury (ie, severe burns). Pyropoikilocytosis has been rarely reported in drug-induced TMA and in severe bacterial bloodstream infections (most commonly Gram-negative bacilli). This patient has received gemcitabine (a known cause of drug-induced TMA) and has a recently diagnosed infection ( $C$ difficile colitis), either of which could be linked to this rare blood smear finding. Both of these syndromes would be treated with supportive care plus avoidance of future gemcitabine.

Transfusion of packed RBCs is indicated given his profound anemia and symptoms of fatigue. One should obtain further testing for cold agglutinins, $\mathrm{PNH}$, and echocardiography to exclude endocarditis. If he were to become critically ill, anuric, or encephalopathic, then one could consider plasma exchange for treatment of TMA and hemoglobin-mediated AKI. Pyropoikilocytosis should be considered the result of drug-induced TMA, severe $C$ difficile colitis, or an occult infection.

The patient was transfused packed RBCs. Because of a concern for an acute TMA such as TTP, both a hematopathologist and the consulting hematology/oncology team reviewed the peripheral blood morphology emergently. $\mathrm{He}$ was given aggressive fluid resuscitation and received $3 \mathrm{~L}$ of IV lactated ringers' solution. An echocardiogram did not show valvular abnormalities. A renal biopsy was contraindicated because of the severe thrombocytopenia.

Given the recently confirmed $C$ difficile colitis along with the findings of pyropoikilocytosis on the peripheral smear, toxin-mediated intravascular hemolysis from systemic $C$ difficile infection became the leading diagnosis. Positing that the $C$ difficile colitis was inadequately treated with oral metronidazole, aggressive treatment for $C$ difficile was initiated with oral vancomycin in addition to intravenous metronidazole. Intravenous metronidazole was included given his elevated creatinine, presence of severe colitis on imaging, and concern he may be at risk for translocation of colonic $C$ difficile or exotoxin into the bloodstream.
Over the course of the next 3 days, the patient's platelet count normalized and his hemoglobin, creatinine, and symptoms of fatigue improved. Blood cultures remained negative. The patient's rapid improvement with antibiotics supported our final diagnosis of toxin-mediated hemolysis caused by a systemic $C$ difficile infection. On follow-up testing after hospital discharge, hemoglobin had returned to prior baseline and there was no recurrent hemolysis. Gemcitabine was considered to be a possible cause of his hemolytic anemia and was not continued in further treatment for his NSCLC.

\section{COMMENTARY}

When evaluating patients with cancer who present with fatigue, hospitalists should consider a broad list of potential causes. The differential should include etiologies directly related to the malignancy, paraneoplastic phenomena, treatment-related complications, and diseases unrelated to cancer. In addition, as the number of medications used for cancer proliferates, hospitalists must take a detailed history of the agents used and be aware of major side effects. Using this information, hospitalists may undertake a targeted approach to diagnostics while searching for a cause of fatigue.

When lab testing reveals profound anemia, hospitalists must consider syndromes that may require emergent management. Anemia can be caused by decreased RBC production, and acute anemia in the absence of clear blood loss suggests hemolysis. Moreover, the combination of elevated LDH and low haptoglobin is quite specific of hemolytic anemia. ${ }^{1,2}$ Once hemolytic anemia is identified, DIC and TMA syndromes (such as TTP) need to be considered. The combination of hemolytic anemia and AKI may indicate a medical emergency and should prompt hospitalists to obtain an urgent peripheral blood smear to help narrow the differential. ${ }^{3}$

The absence of schistocytes on a blood smear does not rule out TTP or HUS, but does argue strongly against these diagnoses. ${ }^{4,5}$ Of note, consultation with a hematopathologist and hematology subspecialist should be done to ensure appropriate and timely review of the peripheral blood smear.

In this case, the blood smear led to a very rare finding of pyropoikilocytosis. The unexpected result should prompt a broader review of the medical history particularly as it relates to the patient's broader symptoms and laboratory abnormalities. Acquired pyropoikilocytosis is a very specific finding known to be associated only with hyperthermal injury (seen in burn patients), drug-induced TMA, and bacterial bloodstream infections, mainly Gram-negative toxins and Clostridioidal infections. ${ }^{6-8}$ In this case, both drug-induced TMA and $C$ difficile infection were considered.

Gemcitabine-induced TMA can occur with either short or long term use of the medication and can be difficult to distinguish from TTP. While both TTP and gemcitabine-induced TMA can cause thrombocytopenia, hemolytic anemia, and schistocytes on a blood smear, the latter causes acute kidney injury more frequently than TTP. In addition, gemcitabineinduced TMA may not lead to severe decrease in ADAMTS13 activity. A kidney biopsy could confirm drug-induced TMA but 
was contraindicated in this case because of the thrombocytopenia. Gemcitabine should not be restarted if this side effect is suspected.

Given the continued rise in $C$ difficile incidence, hospitalists should be aware that $C$ difficile infection can cause extraintestinal illness. 9,10 Although uncommon, these extraintestinal complications are associated with high risk of mortality and frequently occur in those with a history of intestinal injury or inflammation and a concomitant bloodstream infection. ${ }^{10} \mathrm{Re}-$ garding the possibility of $C$ difficile contributing to hemolysis in this case, the patient's low blood counts and hemolysis improved concomitantly with more aggressive treatment of C difficile infection. Although his blood cultures were sterile, C difficile is notoriously difficult to culture. Prior case reports have associated $C$ difficile with intravascular hemolysis, which leads to the possibility that the patient did have a very rare manifestation of this unfortunately common infection. ${ }^{11}$

This case provides an excellent example of a diagnostic pivot point initiated by new information that fundamentally alters a patient's diagnostic journey. Here, the finding of pyroipoikilocytes on peripheral smear led us to alter the differential diagnosis. Such pivots are often "surprises" that cause the care team to stop and reconsider their differential diagnosis because the problem representation has fundamentally changed. This patient's unexpected fiery finding provided a pivot point that led to his effective treatment and recovery.

\section{KEY TEACHING POINTS}

- In evaluating symptomatic cancer patients, providers must consider sequelae of the tumor, paraneoplastic phenomena, and treatment-related complications.
- Hemolytic anemia may represent a life-threatening emergency particularly when accompanied by $A K I$ and requires urgent peripheral blood smear evaluation.

- Acquired pyropoikilocytosis is a specific finding known to be associated only with thermal injury, drug-induced TMA, and bacterial toxin-mediated hemolysis.

Disclosures: The authors have nothing to disclose.

\section{References}

1. Weinzierl EP, Arber DA. The differential diagnosis and bone marrow evaluation of new-onset pancytopenia. Am J Clin Pathol. 2013:139(1):9-29. https:// doi.org/10.1309/AJCP50AEEYGREWUZ

2. Marchand A, Galen RS, Van Lente F. The predictive value of serum haptoglobin in hemolytic disease. JAMA.1980;243(19):1909-1911. https://doi:10.1001/ jama.1980.03300450023014

3. Dhaliwal G, Cornett PA, Tierney LM Jr. Hemolytic anemia. Am Fam Physician. 2004;69(11):2599-2606

4. Joly BS, Coppo P, Veyradier A. Thrombotic thrombocytopenic purpura. Blood. 2017;129(21):2836-2846. https://doi.org/10.1182/blood-2016-10-709857.

5. Jokiranta TS. HUS and atypical HUS. Blood. 2017;129(21):2847-2856. https:// doi.org/10.1182/blood-2016-11-709865

6. Baar S, Arrowsmith DJ. Thermal damage to red cells. J Clin Path. 1970;23(7):572-576. https://doi.org/10.1136/jcp.23.7.572

7. Meinders AJ, Dijkstra I. Massive hemolysis and erythrophagocytosis in severe sepsis. Blood. 2014;124(6):841. https://doi.org/10.1182/ blood-2014-04-565663

8. Mcllwaine K, Leach MT. Clostridium perfringens septicaemia. Br J Haematol. 2013;163(5):549. https://doi.org/10.1111/bjh.12551

9. Evans CT, Safdar N. Current trends in the epidemiology and outcomes of Clostridium difficile infection. Clin Infect Dis. 2015;60 (Supp 2):S66-71. https://doi.org/10.1093/cid/civ140

10. Gupta A, Patel R, Baddour LM, Pardi DS, Khanna S. Extraintestinal Clostridium difficile infections: a single-center experience. Mayo Clin Proc. 2014;89(11):1525-36. https://doi.org/10.1016/j.mayocp.2014.07.012

11. Alvarado AS, Brodsky SV, Nadasdy T, Singh N. Hemolytic uremic syndrome associated with Clostridium difficile infection. Clin Nephrol. 2014;81(4):302-6. https://doi.org/10.5414/CN107691 